\title{
GRB 050410 and GRB 050412: are they really dark gamma-ray bursts?
}

\author{
T. Mineo ${ }^{1}$, V. Mangano ${ }^{1}$, S. Covino ${ }^{2}$, G. Cusumano ${ }^{1}$, V. La Parola ${ }^{1}$, E. Troja ${ }^{1}$, P. Roming ${ }^{3}$, D. N. Burrows ${ }^{3}$, \\ S. Campana ${ }^{2}$, M. Capalbi ${ }^{4}$, G. Chincarini ${ }^{2}$, N. Gehrels ${ }^{5}$, P. Giommi ${ }^{6}$, J. E. Hill ${ }^{5,7}$, F. Marshall ${ }^{5}$, A. Moretti ${ }^{2}$, \\ P. O’Brien ${ }^{8}$, M. Page ${ }^{9}$, M. Perri ${ }^{4}$, P. Romano ${ }^{2}$, B. Sbarufatti ${ }^{1}$, G. Sato ${ }^{5}$, and G. Tagliaferri ${ }^{2}$ \\ 1 INAF - Istituto di Astrofisica Spaziale e Fisica Cosmica di Palermo, via Ugo La Malfa 153, 90146 Palermo, Italy \\ e-mail: teresa.mineo@ifc.inaf.it \\ 2 INAF - Osservatorio Astronomico di Brera, via E. Bianchi 46, 23807 Merate (LC), Italy \\ Penn State University, 525 Davey Lab, University Park, PA 16802, USA \\ ASI, Science Data Center, via G. Galilei, 00044 Frascati, Italy \\ NASA/Goddard Space Flight Center, Greenbelt, MD 20771, USA \\ 6 ASI, Unità Osservazione dell'Universo, Viale Liegi 26, 00198 Roma, Italy \\ 7 Universities Space Research Association, 10211 Wincopin Circle, Suite 500, Columbia, MD, 21044-3432, USA \\ 8 Department of Physics \& Astronomy, University of Leicester, LE1 7RH, UK \\ 9 Mullard Space Science Laboratory, University College of London, Holmbury St Mary, Dorking, Surrey RH5 6NT, UK
}

Received 19 October 2006 / Accepted 17 April 2007

\section{ABSTRACT}

\begin{abstract}
Aims. We present a detailed analysis of the prompt and afterglow emission of GRB 050410 and GRB 050412 detected by Swift for which no optical counterpart was observed.

Methods. We analysed data from the prompt emission detected by the Swift BAT and from the early phase of the afterglow obtained by the Swift narrow field instrument XRT.

Results. The $15-150 \mathrm{keV}$ energy distribution of the GRB 050410 prompt emission shows a peak energy at $53_{-21}^{+40} \mathrm{keV}$. The XRT light curve of this GRB decays as a power law with a slope of $\alpha=1.06 \pm 0.04$. The spectrum is well reproduced by an absorbed power law with a spectral index $\Gamma_{\mathrm{x}}=2.4 \pm 0.4$ and a low energy absorption $N_{\mathrm{H}}=4_{-2}^{+3} \times 10^{21} \mathrm{~cm}^{-2}$ which is higher than the Galactic value. The $15-150 \mathrm{keV}$ prompt emission in GRB 050412 is modelled with a hard $(\Gamma=0.7 \pm 0.2)$ power law. The XRT light curve follows a broken power law with the first slope $\alpha_{1}=0.7 \pm 0.4$, the break time $T_{\text {break }}=254_{-41}^{+79} \mathrm{~s}$ and the second slope $\alpha_{2}=2.8_{-0.8}^{+0.5}$. The spectrum is fitted by a power law with spectral index $\Gamma_{\mathrm{x}}=1.3 \pm 0.2$ which is absorbed at low energies by the Galactic column.

Conclusions. The GRB 050410 afterglow light curve reveals the expected characteristics of the third component of the canonical Swift light curve. Conversely, a complex phenomenology was detected in the GRB 050412 because of the presence of the very early break. The light curve in this case can be interpreted as being the last peak of the prompt emission. The two bursts present tight upper limits for the optical emission, however, neither of them can be clearly classified as dark. For GRB 050410, the suppression of the optical afterglow could be attributed to a low density interstellar medium surrounding the burst. For GRB 050412, the evaluation of the darkness is more difficult due to the ambiguity in the extrapolation of the X-ray afterglow light curve.
\end{abstract}

Key words. gamma rays: bursts - X-rays: bursts - X-rays: individuals: GRB 050410 - X-rays: individuals: GRB 050412

\section{Introduction}

Gamma-Ray Bursts (GRBs), the brightest explosions in the universe, produce emission across the whole electromagnetic spectrum from $\gamma$-rays to radio wavelengths. However, multiwavelength observations have shown that the optical counterpart following the prompt emission is detected in only about $50 \%$ of the well localised events (De Pasquale et al. 2003; Roming et al. 2006; Roming \& Mason 2006).

The paucity of optical detections of GRB afterglows has been explained by invoking different mechanisms (Lazzati et al. 2002; Lamb \& Reichart 2000; Groot et al. 1998; Taylor et al. 1998; Wijers et al. 1998; Totani 1997). More recently, by comparing the Swift optical, X-ray and $\gamma$-ray data sets, Roming et al. (2006) identified a class of optically "dark" GRBs with higher than normal $\gamma$-ray efficiency. A possible mechanism proposed for these GRBs is based on a Poynting flux dominated outflow (Zhang \& Kobayashi 2005) where the transfer of the energy from the fireball to the medium is delayed, leading to the suppression of the reverse shock, likely responsible for the prompt optical emission, and to an apparent high $\gamma$-ray efficiency. A stronger alternative mechanism proposed is a pure non-relativistic hydrodynamical reverse shock (Kobayashi 2000; Nakar \& Piran 2004; Beloborodov 2005; Kobayashi et al. 2005; Uhm \& Beloborodov 2006; McMahon et al. 2006).

The characterisation of optical darkness has been previously based on the upper limit of the optical/NIR afterglow flux (Rol et al. 2005; Filliatre et al. 2005, 2006) or the optical-to-X-ray spectral index (Jakobsson et al. 2004). In particular, if the optical-to-X-ray spectral index, $\beta_{\mathrm{ox}}$, is lower than 0.5 the afterglow should be classified as dark; while for Rol et al. (2005) dark afterglows are those with optical upper limits falling below the extrapolation of the X-ray spectrum to the optical range.

The Swift Gamma-Ray Burst Explorer (Gehrels et al. 2004), successfully launched on 2004 November 20, is a multiwavelength observatory dedicated to the discovery and study 
of GRBs and their afterglows. It carries three instruments: the Burst Alert Telescope (BAT; Barthelmy et al. 2005) and the two narrow-field instruments: the X-Ray Telescope (XRT; Burrows et al. 2005) and the Ultra-Violet Optical Telescope (UVOT; Roming et al. 2005b). Swift's capability of rapidly repointing the spacecraft in a few tens of seconds after a BAT detection, allows a study of the first phases of an afterglow evolution over a broad energy range from optical to X-rays.

In this paper, we present the results on the analysis of the prompt and the afterglow emission of GRB 050410 and GRB 050412 observed by Swift, two bursts for which no optical counterpart was detected.

Errors in the paper are relative to a $90 \%$ confidence level for a single parameter $\left(\Delta \chi^{2}=2.71\right)$. Times are referenced from the BAT trigger, $T_{0}$. The decay and spectral indices are parameterised as follows: $F(v, T) \propto T^{-\alpha} v^{-\beta}$ where $F(v, T)$ $\left(\mathrm{erg} \mathrm{cm}^{-2} \mathrm{~s}^{-1} \mathrm{~Hz}^{-1}\right)$ is the monochromatic flux as a function of time $T$ and frequency $v$; we also use $\Gamma=\beta+1$ as the photon in$\operatorname{dex} N(E) \propto E^{-\Gamma}\left(\mathrm{ph} \mathrm{cm}^{-2} \mathrm{~s}^{-1} \mathrm{keV}^{-1}\right)$ where $N(E)$ is the number of photons at the energy $E$.

\section{Observation}

GRB 050410 triggered BAT at 12:14:25.36 UT (Fenimore et al. 2005). At the time of the first detection, GRB 050410 was within the Swift Earth-limb constraint, therefore, the observatory executed a delayed automated slew to the BAT position and started observing with the narrow-field instruments $\sim 32$ min after the burst. At that time, the X-ray afterglow was too faint for the XRT to produce an on-board centroid, therefore a position was determined during ground processing (La Parola et al. 2005). No new source was detected by UVOT within the BAT and XRT error circles down to a $3 \sigma$ limiting magnitude of $V=19.9, B=21.2$ and $U=20.9$ (Boyd et al. 2005). Ground based follow-up also did not detect any optical counterpart for GRB 050410 down to a limiting magnitude of $R \sim 20.5$ at times after the burst of $T=$ $13.3 \mathrm{ks}$ (Misra et al. 2005), $T=17.5 \mathrm{ks}$ (Ofek \& Lipkin 2005) and $T=20.6 \mathrm{ks}$ (Rumyantsev et al. 2005); and limiting magnitudes of Gunn $i=21.5$ and Gunn $z=20.0$ at $T=56.2 \mathrm{ks}$ after the burst (Cenko \& Fox 2005a). No radio counterpart was detected down to $2 \sigma$ upper limit of $114 \mu \mathrm{Jy}$ (Soderberg 2005a).

The second burst considered in the paper, GRB 050412, was discovered by Swift at 05:44:03 UT (Cummings et al. 2005; Tueller et al. 2005). The observatory executed an automated slew to the BAT position and the XRT and UVOT began taking data $99 \mathrm{~s}$ after the BAT trigger. The position of the X-ray counterpart was derived from the first orbit of data and was given in Mangano et al. (2005). UVOT data revealed no evidence of a fading source in the $5^{\prime \prime}$ radius XRT error circle; the $3 \sigma$ limiting magnitude was $V=19.1$ (Roming et al. 2005a). The Chandra X-ray Observatory observed GRB 050412 with the ACIS for $20 \mathrm{ks}$ on 2005 April 17, when the source was no longer visible in the XRT data. No X-ray source was detected by Chandra and an upper limit on the unabsorbed $0.5-10 \mathrm{keV}$ flux of $3.6 \times$ $10^{-15} \mathrm{erg} \mathrm{cm}^{-2} \mathrm{~s}^{-1}$ was inferred (Berger \& Fox 2005).

Ground based optical follow-up did not detect any counterpart for GRB 050412 at early epochs down to a limiting magnitude of $R \sim 20$ at times after the burst of $T=7.1 \mathrm{~s}$ (Quimby et al. 2005), $T=250 \mathrm{~s}$ (Torii 2005); $T=360 \mathrm{~s}$ (Cenko \& Fox 2005b) and $T=2.5 \mathrm{ks}$, (Berger et al. 2005). The most constraining optical observation was performed with the FOCAS on the Subaru $8.2 \mathrm{~m}$ telescope atop Mauna Kea at $2.3 \mathrm{~h}$ from the burst and it gave a 3-sigma upper limit of $R_{\mathrm{c}}=24.9$ on the afterglow emission (Kosugi et al. 2005). However, a source at
Table 1. Observation $\log$ for the data used in the analysis of GRB 050510 and GRB 050512.

\begin{tabular}{|c|c|c|c|}
\hline \multirow[t]{2}{*}{ Obs. \# } & \multirow{2}{*}{$\begin{array}{c}\text { Start time (UT) } \\
\text { (yyyy-mm-dd hh:mm:ss) }\end{array}$} & \multicolumn{2}{|c|}{ Exposure } \\
\hline & & WT $(s)$ & $\mathrm{PC}(\mathrm{s})$ \\
\hline \multicolumn{4}{|c|}{ GRB 050410} \\
\hline 1 & 2005-04-10 12:46:24 & 9436 & 8635 \\
\hline 2 & 2005-04-12 16:15:47 & - & 4740 \\
\hline 3 & 2005-04-12 00:11:47 & - & 1473 \\
\hline 4 & 2005-04-14 00:22:44 & - & 3184 \\
\hline 5 & 2005-04-15 00:30:44 & - & 10895 \\
\hline 6 & 2005-04-16 00:32:01 & - & 13086 \\
\hline 7 & 2005-04-17 16:45:01 & - & 6418 \\
\hline 8 & 2005-04-19 00:50:42 & - & 9832 \\
\hline 9 & 2005-04-20 00:54:29 & - & 11123 \\
\hline \multirow[t]{2}{*}{10} & 2005-04-21 00:55:58 & - & 1828 \\
\hline & Total exposure (s) & 9436 & 71214 \\
\hline \multicolumn{4}{|c|}{ GRB 050412} \\
\hline 1 & 2005-04-12 05:45:47 & 3019 & 1950 \\
\hline 2 & 2005-04-14 00:04:59 & - & 3719 \\
\hline \multirow[t]{2}{*}{3} & 2005-04-17 09:28:35 & - & 6200 \\
\hline & Total exposure (s) & 3019 & 11869 \\
\hline
\end{tabular}

the centre of the XRT error circle with the magnitude of $R_{\mathrm{c}}=$ $26.0 \pm 0.5$ was detected $7.2 \mathrm{~h}$ after the burst with a second set of observations but because of the marginal detection, it was not possible to determine whether it was a point source or a galaxy (Kosugi et al. 2005). No radio counterpart of GRB 050412 was detected down to $2 \sigma$ upper limit of $38 \mu \mathrm{Jy}$ (Soderberg 2005b).

\section{BAT and XRT data reduction and analysis}

The BAT event data were re-analysed using the standard analysis software $^{1}$ included in the HEAsoft 6.0.4 package. Response matrices were generated with the task BATDRMGEN using the latest spectral redistribution matrices. The BAT background was subtracted using a mask-weighting technique that is only effective up to $150 \mathrm{keV}$.

XRT data were calibrated, filtered and screened with the XRTDAS software package, included in the HEAsoft 6.0.4 package, to produce cleaned photon list files. Only time intervals with the $\mathrm{CCD}$ temperature below $-47 \mathrm{C}^{\circ}$ were selected. Table 1 shows the observation $\log$ for GRB 050510 and GRB 050512.

Standard grade selection, 0-12 for Photon Counting (PC) mode and 0-2 for Window Timing (WT) mode (Hill et al. 2004), was used for both spectral and timing analysis. Ancillary response files for PC and WT spectra were generated with the standard XRTMKARF tool (v 0.5.1) using calibration files from CALDBv.2.3.

WT data were extracted in a rectangular region $40 \times$ 20 pixels along the image strip which included about $96 \%$ of the Point Spread Function (PSF). PC data were extracted from a circular region of 20 pixels radius for observing intervals with a rate lower than 0.2 counts $\mathrm{s}^{-1}$. In time periods with higher rates, the extraction region used was an annulus with an inner radius of 3 pixels and an outer radius of 20 pixels in order to take into account pile-up effects. The two regions included, assuming an average energy of $1.5 \mathrm{keV}, 92 \%$ (circular) and $48 \%$ (annulus) of the PSF, respectively. The WT and PC backgrounds were extracted from regions far from the GRB counterpart and from any sources in the field. WT and PC spectral analysis of both GRBs was performed in the energy range $0.7-10 \mathrm{keV}$ to avoid residual

\footnotetext{
${ }^{1}$ see http://swift.gsfc.nasa.gov/docs/swift/
} 


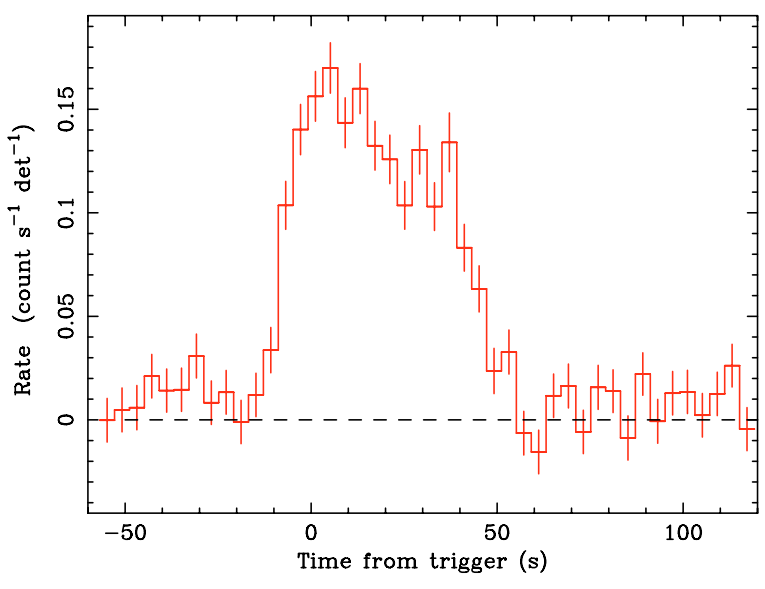

Fig. 1. GRB 050410: BAT light curve in the energy range 15-150 keV. Times are referenced to the first burst trigger (12:14:25.36 UT).

Table 2. Best fit parameters for the spectral analysis in the energy ranges $15-150 \mathrm{keV}$ (BAT) and $0.7-10 \mathrm{keV}$ (XRT) for GRB 050410 and GRB 050412.

\begin{tabular}{|c|c|c|}
\hline & "GRB 050410 & $\overline{\text { GRB } 050412}$ \\
\hline \multicolumn{3}{|c|}{$15-150 \mathrm{keV}$ (BAT) } \\
\hline \multicolumn{3}{|l|}{ Power Law } \\
\hline$\Gamma$ & $1.66 \pm 0.07$ & $0.7 \pm 0.2$ \\
\hline$\chi_{\text {red }}^{2}$ (d.o.f.) & $1.67(36)$ & $0.55(15)$ \\
\hline \multicolumn{3}{|c|}{ Band Model with $\beta_{\text {Band }}=-2.3$} \\
\hline$\alpha_{\text {Band }}$ & $-0.79 \pm 0.09$ & - \\
\hline$E_{\mathrm{p}}(\mathrm{keV})$ & $53_{-21}^{+40}$ & - \\
\hline$\chi_{\text {red }}^{2}$ (d.o.f.) & $1.11(35)$ & - \\
\hline \multicolumn{3}{|c|}{ Cut-off Power Law } \\
\hline$\alpha_{\text {cutoff }}$ & $-0.8 \pm 0.3$ & - \\
\hline$E_{\mathrm{p}}$ & $54_{-17}^{+31}$ & - \\
\hline$\chi_{\text {red }}^{2}$ (d.o.f.) & $1.08(35)$ & \\
\hline
\end{tabular}

\section{$0.7-10 \mathrm{keV}(\mathrm{XRT})$}

\begin{tabular}{lcc} 
Power Law & & \\
$N_{\mathrm{H}}\left(\mathrm{cm}^{-2}\right)$ & $4_{-2}^{+3} \times 10^{21}$ & $2.21 \times 10^{20}$ \\
$\Gamma_{\mathrm{x}}$ & $2.4 \pm 0.4$ & $1.3 \pm 0.2$ \\
$\chi_{\text {red }}^{2}$ (d.o.f. $)$ & $0.64(13)$ & $1.18(17)$ \\
\hline
\end{tabular}

background due to bright Earth contamination and dark current that are more dominant at lower energies.

\subsection{GRB 050410}

GRB 050410 triggered Swift twice as a result of an anomalous time-out of the triggering code (Fenimore et al. 2005). In our analysis, we consider as reference time the time of the first trigger $\left(T_{0}=12: 14: 25.36 \mathrm{UT}\right)$.

The 15-150 keV light curve of the prompt emission, shown in Fig. 1, is characterised by a broad peak with $T_{90}=44 \pm 1 \mathrm{~s}$. The burst shape can be modelled by two Gaussians of the same width $(11 \pm 2 \mathrm{~s})$, the first centered at $5 \pm 1 \mathrm{~s}$ and the second at $32 \pm 2 \mathrm{~s}$.

The $15-150 \mathrm{keV}$ energy distribution of the burst, modelled with a simple power law of photon index $\Gamma=1.66 \pm 0.07$, gave a marginally acceptable fit with a $\chi_{\text {red }}^{2}$ of 1.67 for 36 d.o.f. (see Table 2). Better fits were obtained using a cut-off power law or a Band model (Band et al. 1993) with the high energy photon index, $\beta_{\text {Band }}$, fixed to -2.3 according to the expected value

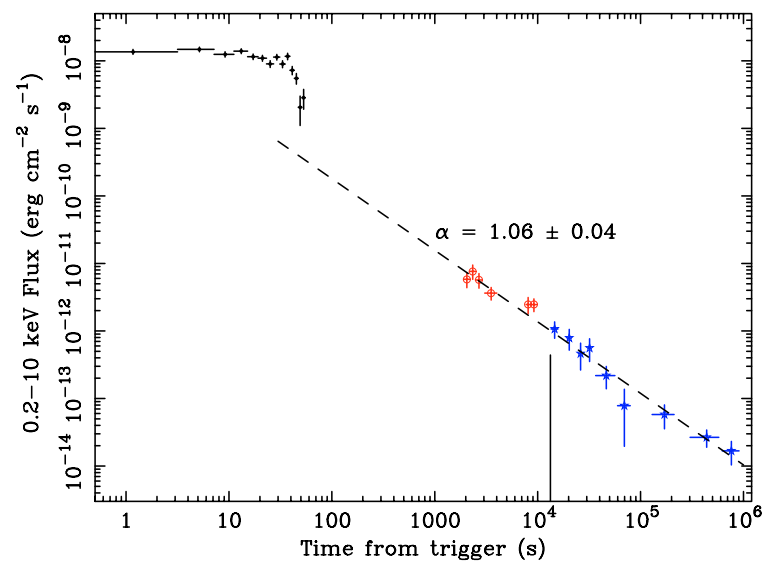

Fig. 2. GRB 050410 light curve. The XRT $0.2-10 \mathrm{keV}$ count rate was converted to flux by applying a conversion factor derived from the spectral analysis. The dashed line represent the best fit model. The BAT light curve was extrapolated to the XRT band by converting the BAT count rate with a factor derived from the Band spectral parameters (see Table 2). The vertical line at $13.3 \mathrm{ks}$ indicate the time of the Misra et al. (2005) optical upper limit.

(Preece et al. 2000). The best fit values of the spectral parameters are shown in Table 2. The improvement with respect to the power law fit is significant for both models, with a chance random probability of $\sim 7 \times 10^{-5}$, assuming that the errors are normally distributed. The fluence in the $15-150 \mathrm{keV}$ energy range was $(4.6 \pm 0.1) \times 10^{-6} \mathrm{erg} \mathrm{cm}^{-2}$.

The XRT observed GRB 050410 for 10 orbits for a total exposure of $\sim 9 \mathrm{ks}$ in WT mode and $\sim 71 \mathrm{ks}$ in PC mode. The position of the burst, determined with astrometry solutions, was $\mathrm{RA}_{\mathrm{J} 2000}=05^{\mathrm{h}} 59^{\mathrm{m}} 13^{\mathrm{s}} .94 \mathrm{Dec}_{\mathrm{J} 2000}=+79^{\circ} 36^{\prime} 11^{\prime \prime} .7$, with an uncertainty of 2'.3 (Butler 2007). These coordinates lie outside the error circle (3".7 radius) of the position derived with the tool XRTCENTROID and $42^{\prime \prime}$ from the BAT position reported by Fenimore et al. (2005).

The GRB 050410 XRT light curve shows a clear decay with time that can be well modelled ( $\chi_{\text {red }}^{2}=0.87$ with 13 d.o.f.) by a single power law with a slope $\alpha=1.06 \pm 0.04$. The light curve, shown in Fig. 2, was converted to flux in the $0.2-10 \mathrm{keV}$ band using a conversion factor derived from the best fit spectral model (see below). In the same figure, the BAT light curve extrapolated to the $0.2-10 \mathrm{keV}$ energy range is also plotted. The extrapolation was obtained by multiplying the $15-150 \mathrm{keV}$ rate by the average rate-to-flux conversion factor for GRB 050410 derived from the Band model parameters.

WT and PC spectra were fitted simultaneously because no spectral evolution with time was detected. The two spectra normalisations were left free to vary, thus accounting for the different average flux levels in the two observing periods. An absorbed power law reproduces well the emission $\left(\chi_{\text {red }}^{2}\right.$ of 0.64 for 13 d.o.f.) with the best fit parameters (see also Table 2) $\Gamma_{\mathrm{x}}=2.4 \pm 0.4$ and $N_{\mathrm{H}}=4_{-2}^{+3} \times 10^{21} \mathrm{~cm}^{-2}$. Note that the absorbing column is non-zero at the 4-sigma level, and the best-fit value of the absorbing column is $\sim 5$ times larger than the Galactic one $\left(7.53 \times 10^{20} \mathrm{~cm}^{-2}\right.$; Dickey \& Lockman 1990).

\subsection{GRB 050412}

The GRB 050412 prompt emission showed a double-peaked structure with the first peak centered at $-1.1 \pm 0.4 \mathrm{~s}$ from the trigger (width of $4.7 \pm 0.6 \mathrm{~s}$ ), and the second fainter and wider 


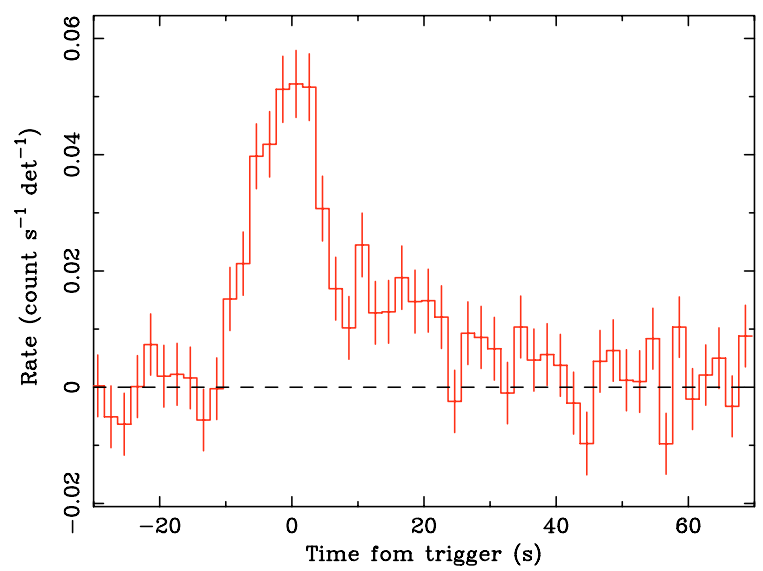

Fig. 3. GRB 050412: BAT light curve in the energy range 15-150 keV.

$(11 \pm 3 \mathrm{~s})$ peak centered at $16 \pm 5 \mathrm{~s}$ (see Fig. 3$)$. The estimated $T_{90}$ in the $15-150 \mathrm{keV}$ energy band was $27 \pm 1 \mathrm{~s}$. The hardness ratio (50-150 keV and $15-50 \mathrm{keV})$ does not show any significant evidence of spectral variation during the burst evolution.

The 15-150 keV energy distribution was well described $\left(\chi_{\text {red }}^{2}=0.55\right.$ for 15 d.o.f.) by a single power law with an hard photon index of $\Gamma=0.7 \pm 0.2$. The $T_{90}$ burst fluence in the $15-150 \mathrm{keV}$ energy range was $(6.3 \pm 0.3) \times 10^{-7} \mathrm{erg} \mathrm{\textrm {cm } ^ { - 2 }}$. A Band model and a cut-off power law were not able to reproduce this spectrum because some of the fitting parameters are not constrained.

The XRT detected a rapidly fading source at $\mathrm{RA}_{\mathrm{J} 2000}=$ $12^{\mathrm{h}} 04^{\mathrm{m}} 25^{\mathrm{s}} .2 \operatorname{Dec}_{\mathrm{J} 2000}=-01^{\circ} 12^{\prime} 00^{\prime} \cdot 4$, with an uncertainty of 4 '. 2 . This position, derived with the tool XRTCENTROID, is 60 '. 8 from the BAT position (Tueller et al. 2005) and it is in agreement with the refined value derived with astrometry technique (Butler 2007).

The XRT light curve is not consistent with a single power law $\left(\chi_{\text {red }}^{2}=2.3\right.$ with 18 d.o.f. $)$. A better fit $\left(\chi_{\text {red }}^{2}=0.7\right.$ with 14 d.o.f.) can be obtained with a broken power law whose best fit parameters are: $\alpha_{1}=0.7 \pm 0.4, \alpha_{2}=2.8_{-0.8}^{+0.5}$ and $T_{\text {break }}=254_{-41}^{+79} \mathrm{~s}$. The GRB 050412 light curve was converted to the $0.2-10 \mathrm{keV}$ flux with a procedure similar to the one used for GRB 050410, and plotted in Fig. 4 together with the BAT light curve extrapolated to the XRT energy range. In the same figure, the $3 \sigma$ upper limit obtained by Chandra is also plotted (Berger \& Fox 2005). The upper limits derived from Swift observations 2 (2005-04-14) and 3 (2005-04-17) are not plotted in the figure, since they are less constraining than the Chandra one.

The first orbit of the GRB 050412 observation is affected by a continuous switching between PC and WT mode (caused by flickering pixels produced by the high CCD temperature and by the bright Earth contamination); thus the two operational mode spectra are almost contemporaneous. The WT spectrum only employed the first $1400 \mathrm{~s}$ of data to avoid a background flare present in the second part of the observation. WT and PC spectra were fitted simultaneously with an absorbed power-law model, leaving the normalisation free in order to take into account differences in the flux levels. The measured absorption column was consistent with the Galactic $\left(2.21 \times 10^{20} \mathrm{~cm}^{-2}\right.$; Dickey \& Lockman 1990) and was fixed to this value. The best fit powerlaw spectral index was $\Gamma_{\mathrm{x}}=1.3 \pm 0.2$ with a reduced $\chi^{2}$ of 1.18 (17 d.o.f.). Results of the spectral analysis are shown in Table 2.

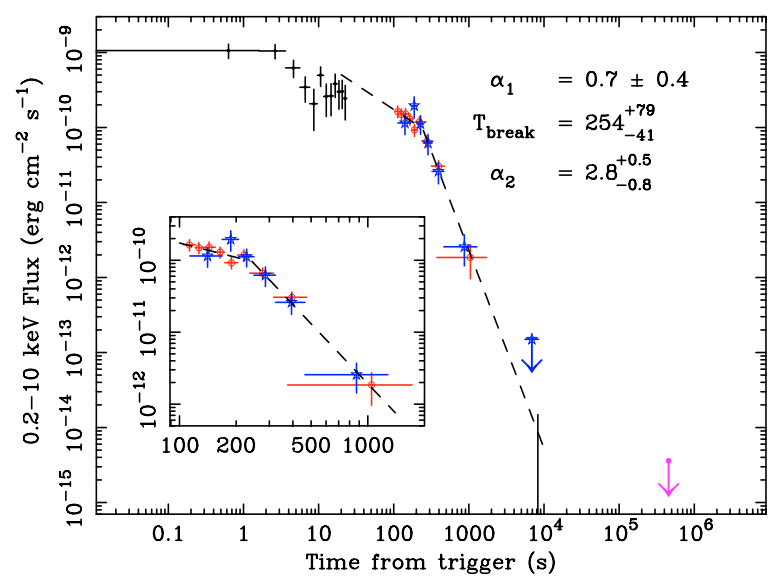

Fig. 4. GRB 050412 light curve. The XRT $0.2-10 \mathrm{keV}$ count rate was converted to flux by applying a conversion factor derived from the spectral analysis. The red circles indicate WT data, the blue stars indicate PC data; the solid line represent the best fit model. The BAT light curve was extrapolated to the XRT band by converting the BAT count rates with the factor derived from the spectral parameters. The $3 \sigma$ upper limit at $4.5 \times 10^{5} \mathrm{~s}$ was obtained by Chandra. The vertical line at $8.3 \mathrm{ks}$ indicate the time of the Kosugi et al. (2005) optical upper limit.

\section{Discussion}

The discussion on the results of GRB 050410 and GRB 050412 analysis is based on two main points: (i) the comparison of the X-ray afterglow light curves with other Swift results, particularly important for GRB 050412 where a very early break ( 250 s) followed by a steep decay $\alpha_{2}=2.8_{-0.8}^{+0.5}$ were detected; (ii) the classification of the two bursts as dark.

\subsection{The $X$-ray light curve}

The X-ray afterglow light curves of many bursts manifest a similar behaviour (O'Brien et al. 2006; Nousek et al. 2006; Willingale et al. 2006). The canonical X-ray light curve has an initial steep decay (usually interpreted as emission from the tail of the prompt GRB), followed by a flatter decay phase that can last up to $10^{5} \mathrm{~s}$ (interpreted as a refreshing of the forward shock), and a final steeper decay phase with power law indices consistent with the values measured before the launch of Swift (Frontera 2003). This canonical light curve is consistent with about $60 \%$ of the Swift afterglows. In many of the nonconforming cases, the first, or the first and the second branches are missing. The lack of detection of these portions of the light curve cannot always be attributed to missing observational data. Moreover, in about half of the afterglows, late X-ray flares, probably due to continued activity of the central engine, are observed (O’Brien et al. 2006; Nousek et al. 2006; Zhang et al. 2006).

The light curve of GRB 050410, starting from about $2000 \mathrm{~s}$ from the trigger, shows a constant decay slope that can be interpreted as the third branch of the canonical light curve. The synchrotron radiation theory applied to the fireball model predicts that the temporal decay index, $\alpha$, of a GRB afterglow and the spectral slope, $\beta$, are linked by relations that depend (i) on the density profile of the external medium (Meszaros \& Rees 1997; Chevalier \& Li 1999); (ii) on the observer's perception of the geometry of the expansion: spherical whenever the expansion velocity corresponds to a Lorentz factor, $\Gamma_{\mathrm{e}}$, such that $\theta_{0}>\Gamma_{\mathrm{e}}^{-1}$, and beamed for $\theta_{0}<\Gamma_{\mathrm{e}}^{-1}$ with $\theta_{0}$ being the half opening angle of the jet (Sari et al. 1999; Rhoads 1999); (iii) on the observational frequency and its relation to the typical synchrotron 
frequency of newly shocked electrons, $v_{m}$, and to the cooling frequency $v_{\mathrm{c}}$ corresponding to a synchrotron cooling time equal to the hydrodynamical expansion time (Sari et al. 1998). In particular, for fireball expansion in a uniform interstellar medium we are usually in the $v_{x}>v_{\mathrm{c}}$ regime and expect $\alpha=(3 p-2) / 4$ and $\beta=p / 2$, with the energy distribution of the electrons, $p$, greater than 2 , before the jet edge effect is manifested. This relation is satisfied by GRB 050410 with a value $p=2.08 \pm 0.05$. This value is lower than $p$ values predicted by numerical simulations (2.2-2.5), but still consistent for particle acceleration at ultraluminous shocks. From this result, we can derive a lower limit of $10 \mathrm{~d}$ to the time after the trigger of any jet break. The XRT light curve of GRB 050410 is therefore consistent with a pre-Swift X-ray afterglow light curve, or with the third branch of the canonical Swift afterglow light curve. The late start of XRT observations ( $2000 \mathrm{~s}$ after the trigger) might have prevented the detection of the previous branches.

According to the typical Swift afterglow behaviour, the early break in the X-ray light curve of GRB 050412 may be interpreted as the flat to steep transition corresponding to the end of the refreshing of the forward shock. However the measured spectral index $\beta_{\mathrm{x}}=0.3 \pm 0.2$ would imply a flat electron energy distribution with $p=0.7 \pm 0.3$. The expected temporal slope, either for spherical expansion in a uniform medium or for a standard isotropic wind model in the $p<2$ and $v_{x}>v_{\mathrm{c}}$ regime, is inconsistent with $\alpha_{2}$.

The values of $\alpha_{1}(0.7 \pm 0.4), \alpha_{2}\left(2.8_{-0.8}^{+0.5}\right)$ and $\beta_{\mathrm{x}}(0.3 \pm 0.2)$ of GRB 050412 fit the closure relations corresponding to a jet break occurring in the $v_{m}<v_{x}<v_{\mathrm{c}}$ regime for $p=\left(2 \beta_{\mathrm{x}}+1\right)=1.7 \pm$ 0.3 with $\alpha_{1}=3\left(2 \beta_{\mathrm{x}}+3\right) / 16$ and $\alpha_{2}=\left(2 \beta_{\mathrm{x}}+7\right) / 4$ according to Dai \& Cheng (2001). An early achromatic break has also been detected in the X-rays and optical light curve for GRB 050801 (Rykoff et al. 2006; Covino et al. 2006), while achromatic breaks usually occur at $T>10^{4}$ s (Blustin et al. 2006; Romano et al. 2006; Soderberg et al. 2006; Bloom et al. 2003; Frail et al. 2001; Covino et al. 2006). However, in the case of GRB 050412, this explanation is very unlikely because such an early time for the temporal break would require a very small value for the jet angle $\left(\theta_{0}<1 \mathrm{deg}\right)$ which is at variance with the beaming angle $\left(\theta_{0}>\right.$ $3 \mathrm{deg}$ ) computed, assuming a peak energy greater than $150 \mathrm{keV}$, from the Amati (2006) and Ghirlanda et al. (2004) relations, that holds for most of the bursts.

Alternatively, the first part of the XRT light curve can be considered as the last peak of the prompt emission, and the decay rate beyond $250 \mathrm{~s}$ can be interpreted as due to curvature effect after an instantaneous turn off of the source (Kumar \& Panaitescu 2000). Within this model the relation that should be satisfied is $\alpha_{2}=\beta_{\mathrm{x}}+2$ which is in agreement with our results. After the final detection at $1 \mathrm{ks}$, the X-ray light curve may start following a typical afterglow decay without violating the later upper limits, however a drop of more than three order of magnitudes in flux within the first $10^{4} \mathrm{~s}$ from the trigger and before the emergence of the afterglow is uncommon to Swift GRBs. It is then possible that GRB 050412 was a burst without afterglow (a naked burst) as GRB 050421 (Godet et al. 2006).

\subsection{Darkness}

GRB 050410 and GRB 050412 do not have identified optical/infrared afterglows, therefore, it is worth considering how dark these bursts are. Both bursts are located in regions with moderate or small Galactic extinction $\left(E_{B-V} \sim 0.11\right.$ and 0.02 for GRB 050410 and GRB 050412, respectively). The available optical upper limits for GRB 050410 are too shallow for any

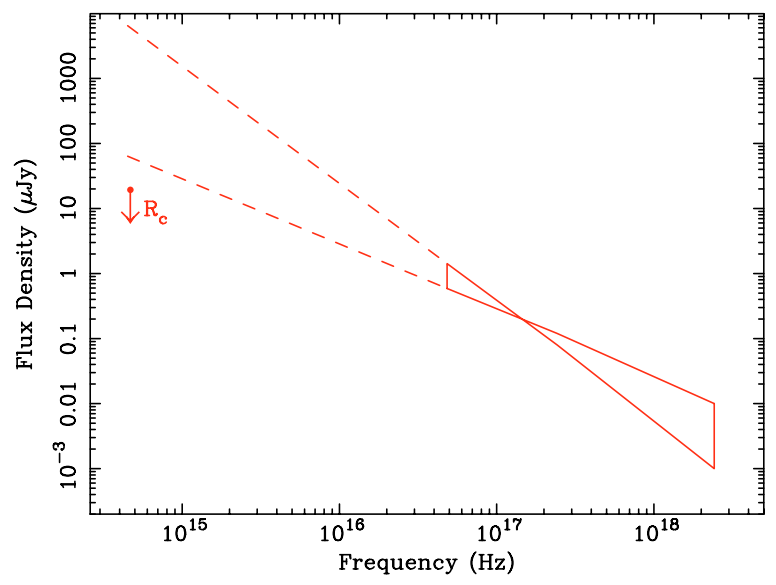

Fig. 5. Spectral energy distribution of the GRB 050410 at 13.3 ks from the burst. The optical measurement is the upper limit reported in Misra et al. (2005); the X-ray spectrum is computed evaluating the $0.2-10 \mathrm{keV}$ flux at the time of the optical point and assuming as spectral model the power law reported in Table 2.

meaningful claim, as shown in Fig. 5 where the broad band spectral energy distribution at the time of the Misra et al. (2005) optical measurement is plotted. The comparison with the X-ray flux ( $\beta_{\mathrm{ox}} \sim 0.8$ computed at $11 \mathrm{~h}$ from the burst) indicates that it was a rather regular burst regards to its gamma-ray to X-ray ratio (see also Roming et al. 2006). However, the $2-10 \mathrm{keV}$ flux computed at $1 \mathrm{~h}\left(2.4 \times 10^{-12} \mathrm{erg} \mathrm{cm}^{-2} \mathrm{~s}^{-1}\right)$ and at $11 \mathrm{~h}(1.9 \times$ $10^{-13} \mathrm{erg} \mathrm{cm}^{-2} \mathrm{~s}^{-1}$ ) shows that this burst has a relatively faint X-ray afterglow (see Fig. 4 in Roming et al. 2006).

A possible interpretation of a low afterglow flux level is a low density medium (Groot et al. 1998; Frail et al. 1999; Taylor et al. 2000). Assuming that the low X-ray flux is due to a low-density circum-burst medium, we derived an estimate of the interstellar medium near the GRB under some reasonable assumptions. From the detected peak energy, $E_{\mathrm{p}}$, in the BAT spectrum, and using the Amati relation (Amati 2006) the values of the energy of the afterglow $E_{\mathrm{a}}\left(\sim 2 \times 10^{51} \mathrm{erg}\right)$ and of the redshift $z(\sim 0.4)$ were inferred. Assuming an electron index of $p=2.5$, taking into account the decay of the afterglow and assuming that the observing frequency is between the peak frequency $v_{\mathrm{m}}$ and the cooling frequency $v_{\mathrm{c}}$, we derived $n<0.01 \mathrm{~cm}^{-3}$. The estimate of $n$, subject to the uncertainty on $z, E_{\mathrm{a}}$ and $p$ (which increases with $z$ and decreases with $E_{\mathrm{a}}$ and $p$ ) is lower than the typical value of GRBs with optical afterglow $\left(\sim 1 \mathrm{~cm}^{-3}\right)$, but similar to values which have already been derived for other GRBs (Panaitescu \& Kumar 2002).

Things are considerably different in the case of GRB 050412: about $2.3 \mathrm{~h}$ after the burst the optical afterglow was not detected with an upper limit of $R_{\mathrm{c}}=24.9$ (Kosugi et al. 2005). In the Rol et al. (2005) Fig. 2 the GRB 050412 representative point would immediately classify it as a very dark burst. However, while any possible optical afterglow for this event is undoubtedly faint already at about $2 \mathrm{~h}$ after the high energy event, to discuss the darkness of the burst a comparison with the detected X-ray flux is mandatory. The X-ray light curve of GRB 050412 shows a break at about $4 \mathrm{~min}$ after the burst and then a steep decay with a temporal index of about 2.8 (see Sect. 3.2). In computing the optical-to-X-ray spectral index at $11 \mathrm{~h}$ after the burst, we may either assume that the optical upper limit went on fading with $T^{-1}$ (as assumed in Jakobsson et al. 2004), or that the optical decay follows the X-ray light curve. In a similar way, the X-ray density flux computed at $11 \mathrm{~h}$ 


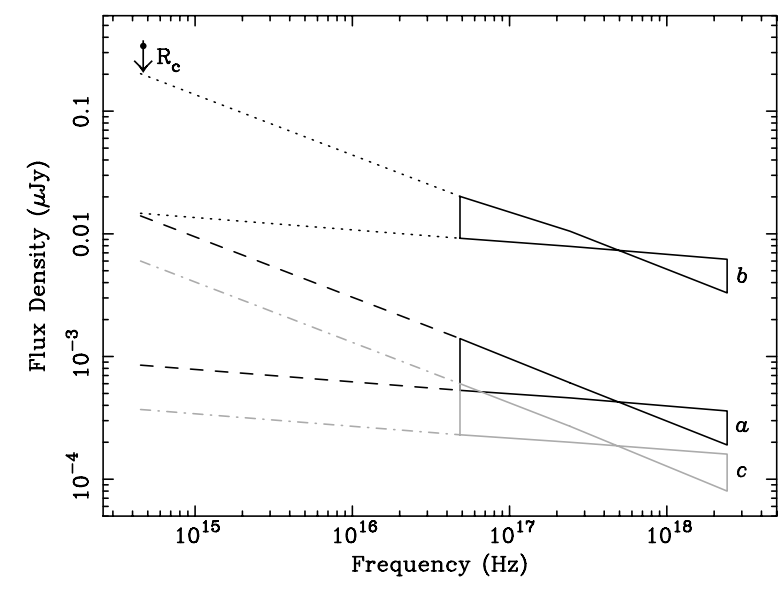

Fig. 6. Spectral energy distribution of the GRB 050412 at $2.3 \mathrm{~h}$ from the burst. The optical measurement is the upper limit reported in Kosugi et al. (2005) The X-ray spectrum labelled with $a$ is computed extrapolating the XRT with a power law with temporal index 2.8 and assuming no spectral variation during the burst evolution (see Table 2). The spectrum labelled with $b$ assumes that the X-ray light curve become flat with a flux value equal to the upper limit measured by XRT at $7 \mathrm{ks}$ from the burst; the curve labelled with $c$ is computed assuming that the light curve become flat at the level of the Chandra upper limit (Berger \& Fox 2005).

from the burst can be evaluated from the extrapolation of the decay curve, or assuming that, at that time, the burst flux reached the level of the Chandra upper limit. The resulting optical-to-X-ray spectral index $\beta_{\mathrm{ox}}$ would not clearly identify this event as dark because it ranges from $\sim 0.5$ to $\sim 1$ depending on how the optical and X-ray flux extrapolation are performed. Of course, the brighter the extrapolated X-ray flux, the more convincing is the classification of GRB 050412 as a dark event.

Figure 6 presents the optical upper limit by Kosugi et al. (2005) at $2.3 \mathrm{~h}$ from the trigger together with the X-ray spectrum computed extrapolating the XRT flux with the measured decay rate $\left(\alpha_{2}=2.8\right)$ and assuming no spectral variation with time (see label $a$ ). In the same figure, the X-ray spectrum is also computed assuming that the flux at $2.3 \mathrm{~h}$ was as high as the upper limit measured by XRT at $7 \mathrm{ks}$ from the burst (see label $b$ ) and that the light curve became flat at the level of the Chandra upper limit (see label $c$; Berger \& Fox 2005). However, we have seen in Sect. 4.1 that the most likely interpretation of the XRT light curve of GRB 050412 is not an afterglow, but the tail of the prompt emission from curvature effect. If this is the case, the possible underlying $\mathrm{X}$-ray afterglow at $2.3 \mathrm{~h}$ is expected to be softer than the measured spectrum (with a photon index $\Gamma \sim 2$ ). This would make the X-ray spectra in Fig. 6 steeper, bringing the expected optical emission well above the observed upper limit, but it would not lead to a firm conclusion about the darkness of GRB 050412.

As last point, looking at Fig. 3 in Roming et al. (2006) both GRB 050410 and GRB 050412 appear fairly normal considering the X-ray flux at $1 \mathrm{~h}$ compared to their prompt $\gamma$-ray flux.

\section{Conclusion}

We have presented a detailed analysis of the prompt and afterglow emission of GRB 050410 and GRB 050412 detected by Swift. For either burst no optical counterparts were detected. Results of the analysis can be summarised as follow:

- The prompt emission lasted $44 \pm 1 \mathrm{~s}$ with a $15-150 \mathrm{keV}$ fluence of $4.6 \pm 0.1 \times 10^{-6} \mathrm{erg} \mathrm{cm}^{-2}$ for the first burst
(GRB 050410) and $27 \pm 1 \mathrm{~s}$ with a 15-150 keV fluence of $(6.3 \pm 0.3) \times 10^{-7} \mathrm{erg} \mathrm{cm}^{-2}$ for the second burst (GRB 050412). The 15-150 keV average energy distribution of the GRB 050410 emission was fitted by a Band model with the peak energy at $53_{-21}^{+40} \mathrm{keV}$ and a low energy slope of $-0.79 \pm 0.09$ after fixing the high energy slope to -3 . The GRB 050412 15-150 keV emission was modelled with a hard $(\Gamma=0.7 \pm 0.2)$ power law suggesting a peak energy above BAT energy range.

- The GRB 050410 XRT light curve can be modelled with a single power law with a slope of $\alpha=1.06 \pm 0.04$. The average spectrum is reproduced by an absorbed power law with a spectral index $\Gamma_{\mathrm{x}}=2.4 \pm 0.4$ and low energy absorption $N_{\mathrm{H}}=4_{-2}^{+3} \times 10^{21} \mathrm{~cm}^{-2}$ which is higher than the galactic value.

The GRB 050412 XRT light curve follows a broken power law with the first slope $\alpha_{1}=0.7 \pm 0.4$, the break time $T_{\text {break }}=254_{-41}^{+79} \mathrm{~s}$ and the second slope $\alpha_{2}=2.8_{-0.8}^{+0.5}$. The average spectrum was fitted by a power law with a spectral index $\Gamma_{\mathrm{x}}=1.3 \pm 0.2$ and absorbed at low energies by a column consistent with the Galactic $\left(N_{\mathrm{H}}=2.21 \times 10^{20} \mathrm{~cm}^{-2}\right)$.

- The GRB 050410 afterglow light curve manifests the expected characteristics of the third component of the canonical Swift light curve and can be interpreted as that X-ray afterglow of a spherical fireball expanding in a uniform medium. In contrast, a rather complex phenomenology was detected in the GRB 050412 X-ray light curve because of a very early break ( $\sim 250 \mathrm{~s})$. A possible explanation for the observed phenomenology suggests the detection of a tail of the prompt emission.

- Upper limits exist for the afterglows of both bursts in the optical, and the upper limit is quite severe in the case of GRB 050412. However, neither burst can be clearly classified as a dark burst according to the definition given by Jakobsson et al. (2004). GRB 050410 has a $\beta_{\mathrm{ox}}=0.8$ and the suppression of the optical afterglow could be attributed to a low density of the interstellar medium surrounding the burst. For the second burst, the proper evaluation of the $\beta_{\mathrm{ox}}$ is quite difficult due to the ambiguity in the extrapolation of the X-ray light curve.

Acknowledgements. This work is supported at INAF by ASI grant I/R/039/04 and by MIUR grant 2005025417, at Penn State by NASA contract NAS5-00136 and at the University of Leicester by PPARC. We gratefully acknowledge the contributions of dozens of members of the XRT and UVOT team at OAB, PSU, UL, GSFC, ASDC, and MSSL and our subcontractors, who helped make this mission possible.

\section{References}

Amati, L. 2006, MNRAS, 372, 233

Band, D., Matteson, J., Ford, L., et al. 1993, ApJ, 413, 281

Barthelmy, S. D., Barbier, L. M., Cummings, J. R., et al. 2005, Space Sci. Rev., 120,143

Beloborodov, A. M. 2005, ApJ, 618, L13

Berger, E., \& Fox, D. B. 2005, GRB Circ. Network, 3291, 1

Berger, E., Morrell, N., Schmidt, B., Fox, D., \& Price, P. 2005, GRB Coordinates Network, 3239, 1

Bloom, J. S., Frail, D. A., \& Kulkarni, S. R. 2003, ApJ, 594, 674

Blustin, A. J., Band, D., Barthelmy, S., et al. 2006, ApJ, 637, 901

Boyd, P., Holland, S. T., Still, M., et al. 2005, GRB Circular Network, 3230, 1

Burrows, D. N., Hill, J. E., Nousek, J. A., et al. 2005, Space Sci. Rev., 120, 165 Butler, N. R. 2007, AJ, 133, 1027

Cenko, S. B., \& Fox, D. B. 2005a, GRB Coordinates Network, 3231, 1

Cenko, S. B., \& Fox, D. B. 2005b, GRB Coordinates Network, 3242, 1

Chevalier, R. A., \& Li, Z.-Y. 1999, ApJ, 520, L29

Covino, S., Malesani, D., Tagliaferri, G., et al. 2006, ArXiv Astrophysics e-prints 
Cummings, J., Barbier, L., Barthelmy, S., et al. 2005, GRB Circ. Network, 3237, 1

Dai, Z. G., \& Cheng, K. S. 2001, ApJ, 558, L109

De Pasquale, M., Piro, L., Perna, R., et al. 2003, ApJ, 592, 1018

Dickey, J. M., \& Lockman, F. J. 1990, ARA\&A, 28, 215

Fenimore, E., Barbier, L., Barthelmy, S., et al. 2005, GRB Circular Network, 3219,1

Filliatre, P., D'Avanzo, P., Covino, S., et al. 2005, A\&A, 438, 793

Filliatre, P., Covino, S., D' Avanzo, P., et al. 2006, A\&A, 448, 971

Frail, D. A., Kulkarni, S. R., Bloom, J. S., et al. 1999, ApJ, 525, L81

Frail, D. A., Kulkarni, S. R., Sari, R., et al. 2001, ApJ, 562, L55

Frontera, F. 2003, Ch. J. Astron. Astrophys., 3, 439

Gehrels, N., Chincarini, G., Giommi, P., et al. 2004, ApJ, 611, 1005

Ghirlanda, G., Ghisellini, G., \& Lazzati, D. 2004, ApJ, 616, 331

Godet, O., Page, K. L., Osborne, J. P., et al. 2006, A\&A, 452, 819

Groot, P. J., Galama, T. J., Vreeswijk, P. M., et al. 1998, ApJ, 502, L123

Hill, J. E., Burrows, D. N., Nousek, J. A., et al. 2004, in X-Ray and Gamma-Ray Instrumentation for Astronomy XIII, ed. K. A. Flanagan, \& O. H. W. Siegmund, Proc. SPIE, 5165, 217

Jakobsson, P., Hjorth, J., Fynbo, J. P. U., et al. 2004, ApJ, 617, L21

Kobayashi, S. 2000, ApJ, 545, 807

Kobayashi, S., Zhang, B., Meszaros, P., \& Burrows, D. N. 2005,

[arXiv: astro-ph/0506157]

Kosugi, G., Kawai, N., Aoki, K., et al. 2005, GRB Circ. Network, 3263, 1

Kumar, P., \& Panaitescu, A. 2000, ApJ, 541, L51

La Parola, V., Mangano, V., Mineo, T., et al. 2005, GRB Circ. Network, 3218, 1

Lamb, D. Q., \& Reichart, D. E. 2000, ApJ, 536, 1

Lazzati, D., Rossi, E., Covino, S., Ghisellini, G., \& Malesani, D. 2002, A\&A, 396, L5

Mangano, V., Capalbi, M., Pagani, C., et al. 2005, GRB Coordinates Network, 3253, 1

McMahon, E., Kumar, P., \& Piran, T. 2006, MNRAS, 366, 575

Meszaros, P., \& Rees, M. J. 1997, ApJ, 476, 232

Misra, K., de Ugarte Postigo, A., Jelinek, M., et al. 2005, GRB Coordinates Network, 3226, 1

Nakar, E., \& Piran, T. 2004, MNRAS, 353, 647

Nousek, J. A., Kouveliotou, C., Grupe, D., et al. 2006, ApJ, 642, 389

O'Brien, P. T., Willingale, R., Osborne, J., et al. 2006, ApJ, 647, 1213

Ofek, E. O., \& Lipkin, Y. 2005, GRB Coordinates Network, 3225, 1
Panaitescu, A., \& Kumar, P. 2002, ApJ, 571, 779

Preece, R. D., Briggs, M. S., Mallozzi, R. S., et al. 2000, ApJS, 126, 19

Quimby, R. M., Rykoff, E. S., \& Schaefer, B. E. 2005, GRB Coordinates Network, 3238, 1

Rhoads, J. E. 1999, ApJ, 525, 737

Rol, E., Wijers, R. A. M. J., Kouveliotou, C., Kaper, L., \& Kaneko, Y. 2005, ApJ, 624,868

Romano, P., Moretti, A., Banat, P. L., et al. 2006, A\&A, 450, 59

Roming, P., Mangano, V., Holland, S. T., et al. 2005a, GRB Circ. Network, 3249, 1

Roming, P. W. A., Kennedy, T. E., Mason, K. O., et al. 2005b, Space Sci. Rev., 120, 95

Roming, P. W. A., \& Mason, K. O. 2006, in American Institute of Physics Conference Series, ed. S. S. Holt, N. Gehrels, \& J. A. Nousek, 224

Roming, P. W. A., Schady, P., Fox, D. B., et al. 2006, ApJ, accepted [arXiv: astro-ph/0509273]

Rumyantsev, V., Biryukov, V., \& Pozanenko, A. 2005, GRB Coordinates Network, 3229, 1

Rykoff, E. S., Mangano, V., Yost, S. A., et al. 2006, ApJ, 638, L5

Sari, R., Piran, T., \& Halpern, J. P. 1999, ApJ, 519, L17

Sari, R., Piran, T., \& Narayan, R. 1998, ApJ, 497, L17

Soderberg, A. M. 2005a, GRB Coordinates Network, 3223, 1

Soderberg, A. M. 2005b, GRB Circ. Network, 3277, 1

Soderberg, A. M., Berger, E., Kasliwal, M., et al. 2006, ApJ, 650, 261

Taylor, G. B., Bloom, J. S., Frail, D. A., et al. 2000, ApJ, 537, L17

Taylor, G. B., Frail, D. A., Kulkarni, S. R., et al. 1998, ApJ, 502, L115

Torii, K. 2005, GRB Coordinates Network, 3245, 1

Totani, T. 1997, ApJ, 486, L71

Tueller, J., Barbier, L., Barthelmy, S., et al. 2005, GRB Circular Network, 3251, 1

Uhm, Z., \& Beloborodov, A. M. 2006, in American Institute of Physics Conference Series, ed. S. S. Holt, N. Gehrels, \& J. A. Nousek, 189

Wijers, R. A. M. J., Bloom, J. S., Bagla, J. S., \& Natarajan, P. 1998, MNRAS, 294, L13

Willingale, R., O’Brien, P. T., Osborne, J. P., et al. 2006, ArXiv Astrophysics e-prints

Zhang, B., Fan, Y. Z., Dyks, J., et al. 2006, ApJ, 642, 354

Zhang, B., \& Kobayashi, S. 2005, ApJ, 628, 315 